\title{
Aplicación de un modelo híbrido de aprendizaje basado en problemas como estrategia de evaluación e interrelación "multiasignaturas"
}

\author{
Esteve Llargués, Xavier Herranz, Lluís Sánchez, Esther Calbo, Montserrat Virumbrales
}

Resumen. Se ha diseñado e implementado un modelo 'híbrido' de aprendizaje basado en problemas (ABP) como estrategia de enseñanza, evaluación e interrelación de las asignaturas de 'Fisiopatogenia' y 'Semiología' del grado de medicina de la Universitat Internacional de Catalunya. El modelo hibrido ha sido diseñado sobre la plataforma de tecnología de la información Moodle que facilita su desarrollo con menos recursos. El ABP implementado funciona como una actividad integradora que trata de fomentar el desarrollo de competencias transversales y el pensamiento complejo, en un entorno de aprendizaje activo. Promueve en el estudiante la construcción de su propio conocimiento para el desarrollo de competencias como el razonamiento clínico y la toma de decisiones, al tiempo que posibilita la integración de los conocimientos procedentes de distintas asignaturas. Los resultados de este trabajo han reflejado un alto grado de satisfacción del alumnado con la metodología fundamentada en el ABP, habiéndose logrado una participación activa en el proceso enseñanzaaprendizaje.

Palabras clave. Aprendizaje basado en problemas (ABP). Grado de medicina.

Application of a hybrid model of problem-based learning as a strategy for assessment and the integration of "multiple subjects"

Summary. A 'hybrid' model of problem-based learning (PBL) was designed and implemented as a teaching strategy, assessment and means to integrate knowledge related to the subjects 'Physiopathogenesis' and 'Semiotics' of Medicine in the Universitat Internacional de Catalunya. The hybrid model was designed within the e-learning platform, Moodle, which facilitates the development of PBL methodology but with fewer resources. PBL functions as an integrative activity which seeks to promote development of generic skills and complex thought in an active learning environment. PBL encourages students to construct their own knowledge for the development of skills such as clinical reasoning and decision-making, while enabling the integration of knowledge from different subjects. The outcomes of this project indicate a high degree of student satisfaction with this PBL-based methodology as has been manifested by the achievement of active participation in the teaching-learning process.

Key words. Medicine degree. Problem-based learning (PBL).

\section{Introducción}

La educación universitaria está en constante evolución para adaptarse a los cambios de la sociedad. En el año 2007, el Plan de Bolonia impulsado por los países de la Unión Europea marcó un punto de inflexión en este proceso. El Plan de Bolonia enfatiza el concepto de 'enseñar a aprender', lo que hace necesaria una educación más integral, basada en la adquisición de competencias y donde los estudiantes pasan a ser el motor de su aprendizaje. Estos cambios han comportado una revisión del diseño de los programas universitarios y una reducción de las clases magistrales, dando paso a otras modalidades de aprendizaje activo e integrado: métodos del caso, laboratorio de habilidades, prácticas clínicas tuteladas, seminarios, entre otros. En este escenario, el profesor pasa de ser un orador a tutorizar el proceso de aprendizaje del alumno [1].

\section{Modelo de aprendizaje basado en problemas (ABP)}

El aprendizaje basado en problemas (ABP) es una metodología de aprendizaje activo y autodirigido que ha ido adquiriendo adeptos en los últimos años, aunque su inicio se remonta a los años sesenta en la
Hospital General de Granollers (E. Llargués). Hospital Universitari Mútua de Terrassa (E. Calbo). Hospital General de Catalunya (X. Herranz, L. Sánchez). Grupo de Investigación e Innovación Docente en Salud (X. Herranz, L. Sánchez, M. Virumbrales). Universitat Internacional de Catalunya (E. Llargués, X. Herranz, L. Sánchez, E. Calbo, M. Virumbrales). Sant Cugat del Vallès, Barcelona, España.

Correspondencia:

Dr. Esteve Llargués Rocabruna. Facultad de Medicina y Ciencias de la Salud. Universitat Internacional de Catalunya. Campus Sant Cugat. Josep Trueta, s/n. E-08195 Sant Cugat del Vallès (Barcelona).

Fax: +34935042001.

E-mail:

ellargues@uic.es

Conflicto de intereses: No declarado.

Competing interests: None declared.

(C) 2015 FEM 
facultad de medicina de la Universidad McMaster (Canadá) [2].

Barrows define el ABP como 'un método de aprendizaje basado en el principio de usar problemas como punto de partida para la adquisición e integración de los nuevos conocimientos' [3].

Desde su origen hasta hoy en día, el ABP ha ido evolucionando y adaptándose a las necesidades de las diferentes áreas y centros universitarios en los que se ha adoptado, lo que ha conllevado numerosas variaciones con respecto a la propuesta original, si bien su finalidad sigue siendo que el estudiante aprenda a buscar, analizar, utilizar la información e integrar el conocimiento a partir de un escenario en el que se le presenta una situación clínica [4-6].

El ABP se fundamenta en tres pilares: la definición de un contexto de aprendizaje, el trabajo en grupos reducidos de estudiantes y la tutorización del profesor. Se trata de un modelo de enseñanzaaprendizaje en el cual el estudiante asume la responsabilidad en el proceso, siendo el protagonista [7].

Esta modalidad de aprendizaje aporta una especial motivación a los estudiantes estimulados por el desafío que supone el estudio de un problema y su posterior resolución. El ABP fomenta el autoaprendizaje, el trabajo en grupo y el aprendizaje colaborativo, estimula el pensamiento crítico y el razonamiento clínico, elaborado a partir de la integración de los conocimientos, y favorece el desarrollo de competencias profesionales. El proceso de estudio con ABP ayuda a mejorar habilidades, como la capacidad de resolver problemas, aprender a transferir conocimiento a nuevas situaciones y adquirir mejores competencias en el ámbito social y cognitivo $[8,9]$.

A pesar de las excelencias del ABP, esta modalidad de estudio no ha tenido la implantación que podría esperarse [10]. Esta situación obedece a las dificultades que comporta la implantación del ABP tal como se concibió en su formato original. La metodología del ABP comporta una mayor dedicación de tiempo del profesor comparado con el método tradicional (tres o cuatro veces más), lo que cuestiona la viabilidad económica del método, especialmente en momentos de 'contención de gastos' o 'escasez de recursos.' Tratando de minimizar las limitaciones fundamentalmente de recursos, tanto humanos como físicos, se vienen desarrollando e implementando modelos de ABP que podríamos llamar 'híbridos' [11].

En el desarrollo curricular del grado de medicina, en la Universitat Internacional de Catalunya (UIC), se contemplan diferentes metodologías de enseñanza-aprendizaje, teniendo un peso predominante las de carácter integrador. En el tercer curso se ubican, entre otras, las asignaturas de 'Fisiopatogenia' y 'Semiología. Las metodologías de enseñanza-aprendizaje empleadas en el desarrollo de ambas asignaturas son: clases magistrales, método del caso, laboratorio de habilidades y prácticas clínicas en planta de hospitalización de medicina interna. Por lo que respecta al sistema de evaluación, éste tiene un componente formativo y otro sumativo. Los instrumentos que se emplean a tal fin son: exámenes de conocimientos con preguntas de respuesta múltiple, evaluación clínica objetiva estructurada (ECOE) y portafolio. En el curso 2012-2013, el ABP se utilizó como herramienta de aprendizaje y también como instrumento de la evaluación sumativa.

El propósito de este trabajo es describir el modelo de ABP implementado en las asignaturas de ' $\mathrm{Fi}$ siopatogenia' y 'Semiología' (ABP-FS), de tercer curso del grado de medicina de la UIC. El modelo desarrollado consistía en adaptar y aplicar la metodología del ABP a nuestra realidad.

\section{Objetivos del ABP-FS}

En la aplicación del ABP-FS se estableció como objetivo el desarrollo de las siguientes competencias genéricas:

- Capacidad de análisis y síntesis.

- Razonamiento crítico.

- Aprendizaje autónomo.

- Capacidad de gestión de la información.

- Trabajo en equipo.

- Comunicación oral y escrita en lengua nativa.

\section{Sujetos y métodos}

\section{Participantes}

Se trabajó con una muestra no probabilística, de conveniencia, formada por 74 alumnos de tercer curso del grado de medicina de la UIC (37 hombres y 37 mujeres), con un rango de edad de 20-27 años (media: 21 años), con los cuales se implementó el ABP-FS como estrategia metodológica de enseñanza-aprendizaje e instrumento de evaluación.

Todos los participantes estaban matriculados en las asignaturas de 'Fisiopatogenia' y 'Semiología'. Estas asignaturas comparten contenidos y los desarrollan de forma complementaria.

\section{Instrumentos}

Se elaboró un cuaderno-guía del ABP en el que se explicaba en qué consistía, objetivos y competen- 
cias a trabajar, metodología de trabajo, incluyendo el rol de alumno tanto individual como grupal, el rol de tutor, el proceso de evaluación y el calendario de trabajo. Además, en este cuaderno se presentaba un caso-problema que, a modo de ejemplo, era analizado y resuelto siguiendo los pasos secuenciales propuestos para el desarrollo del ABP-FS.

Asimismo, se diseñó una encuesta de satisfacción del estudiante respecto del ABP-FS. El cuestionario incluía siete ítems valorados mediante una escala tipo Likert, con cinco opciones de respuesta, de 1 (nada satisfecho/completamente en desacuerdo) a 5 (completamente satisfecho/completamente de acuerdo), y se evaluó el grado de satisfacción de los estudiantes respecto a los recursos proporcionados, la utilidad para el desarrollo del razonamiento clínico, la utilidad para la mejora de la capacidad de análisis, el trabajo en grupo, el tiempo y esfuerzo requeridos, la utilidad para la integración de conocimientos relacionados con las dos asignaturas implicadas y el grado de satisfacción global. El cuestionario también incluía dos ítems en los que se solicitaba a los encuestados que señalasen tres ventajas y tres dificultades encontradas para el desarrollo de la actividad.

\section{Tutores}

Un total de cuatro docentes, comunes a las asignaturas de 'Fisiopatogenia' y 'Semiología', participaron en el diseño y la ejecución del ABP-FS, asumiendo cada profesor el rol de tutor de uno de los casos-problema planteados.

$\mathrm{Su}$ papel fue, por un lado, elaborar los problemas, diseñar los distintos documentos de evaluación del proceso de ABP y una serie de preguntas apropiadas para favorecer que los alumnos recopilasen la información adecuada de manera precisa; por otro, orientar a los alumnos para que alcanzaran los objetivos de aprendizaje y evaluar el trabajo realizado por éstos.

Las sesiones tutorizadas se realizaron a través de la plataforma virtual, salvo una primera que se llevó a cabo con todos los alumnos participantes en un aula. El número de tutorías virtuales podía variar en función de las necesidades de los alumnos o miembros de los grupos, de las percepciones del tutor encargado de guiarlos en cada uno de los problemas, y del grado de dificultad del tema a trabajar.

Las múltiples herramientas disponibles en la plataforma virtual Moodle (wiki, foros, tareas, mensajes) han facilitado la implementación del ABP-FS, resultando muy útil con un número elevado de alumnos. Así, los foros permiten el seguimiento de las actividades del grupo; a través de los mensajes se pueden atender las dudas que surgen a los participantes, y en todo momento el tutor puede identificar la actividad o participación durante el semestre por parte de los alumnos.

Se promovió la resolución de las dudas o cuestiones planteadas por los alumnos lo antes posible, intentando que el plazo de respuesta no sobrepasase las 48 horas. La utilización de recursos virtuales facilitó la labor docente porque el profesor pudo dar respuesta a los alumnos en cualquier momento del día, sin tener que prefijar para ello un horario de consulta, lo que favorecía una mejor gestión de las cargas de trabajo.

\section{Procedimiento de ejecución}

Para el desarrollo del ABP-FS se constituyeron 10 subgrupos de trabajo con 7-8 integrantes. La actividad se llevó a cabo durante el primer semestre del curso (16 semanas lectivas), de septiembre de 2012 a enero de 2013.

Los temas en los que se centraron los casos-problemas planteados ('fiebre', 'síndrome confusional', 'edemas' y 'disnea') fueron consensuados por el equipo docente de las asignaturas.

El procedimiento consistió, en primer lugar, en dedicar al grupo completo (74 alumnos) una sesión, al inicio del semestre, de 30 minutos explicando los objetivos y la metodología de la práctica ABP-FS. A la finalización de esta sesión se entregó el cuadernoguía con las normas e instrucciones, así como el caso modelo resuelto con los pasos a seguir en su desarrollo (Tabla I).

Trascurridas dos semanas se publicaron, a través de la plataforma virtual Moodle, los cuatro casosproblemas planteados, acompañados de preguntas de ayuda para orientar el proceso de análisis de éstos, tras lo cual se llevaron a cabo los cuatro pasos de los que consta el modelo de ABP híbrido propuesto:

- Q1. Análisis, ordenación y priorización de la información.

- Q2. Investigación y 'lluvia de ideas' o formulación de hipótesis.

- Q3. Solicitud de información clínica y exploraciones complementarias que puedan ayudar a argumentar la hipótesis.

- Q4. Resolución.

Estos pasos se desarrollan en cuatro escenarios de trabajo: clase completa, trabajo individual sin tutor, trabajo grupal sin tutor y tutorías virtuales solicitadas, de modo individual o grupal, para clarificar conceptos y orientar el proceso o discusión de los casos. 
Tabla I. Pasos establecidos en el desarrollo del aprendizaje basado en problemas en 'Fisiopatogenia' y 'Semiología' (ABP-FS).

Q1. Análisis, ordenación y priorización de la información clínica del ABP

(de la semana 4 a la semana 6 del semestre)

Se analiza el caso y se listan los datos clínicos del caso clínico para realizar una primera aproximación al juicio clínico

Q2. Lluvia de ideas: formulación de hipótesis a partir de los conocimientos previos, del estudio y de la aportación de las clases de la asignatura (de la semana 7 a la semana 10 del semestre)

Se exponen las hipótesis de diagnóstico que den respuesta a la situación clínica del

Trabajo caso. El punto de partida son los conocimientos previos, que se complementan con el

individual proceso de investigación (búsqueda de información) y los conocimientos que adquiere el estudiante en el curso de la asignatura. Con esta información debe verificar y razonar las hipótesis descritas en un primer momento, o las nuevas hipótesis que surjan a raíz del estudio del caso

Aportación de los resultados del estudio individual -Q1 y Q2, información clínica analizada e hipótesis- a la plataforma Moodle (semana 10 del semestre)

Esta información es analizada por el tutor-responsable del caso y forma parte de la evaluación

Contrastar y someter a discusión la información aportada por los miembros del grupo de trabajo. El grupo repite el paso Q1. Contrastar y someter a discusión las hipótesis aportadas por los miembros del grupo de trabajo. El grupo repite el paso Q2. Aportación de los resultados del estudio grupal-Q1 y Q2, información clínica analizada e hipótesis-a la plataforma Moodle (semanas 11 y 12 del semestre)

Síntesis de los nuevos conocimientos. Verificar hipótesis e identificar objetivos alcanzados

Q3. Demanda de datos clínicos y exploraciones complementarias por parte del grupo de trabajo Los estudiantes tienen una fecha límite para la solicitud de información (semana 12 del semestre)

Se solicita información clínica: datos de historia clínica-anamnesis o exploración física que aporten información para argumentar las hipótesis de trabajo. La solicitud de los

Trabajo datos de la exploración debe argumentarse indicando los resultados esperados o la

grupal interpretación de éstos

Se solicitan exploraciones complementarias que puedan aportar información para argumentar las hipótesis de trabajo. La solicitud de las pruebas debe argumentarse explicando los resultados esperados

El tutor-responsable del caso analiza la información solicitada y remite los datos al grupo (semana 13 del semestre)

Esta información (Q1, Q2 y Q3) es analizada por el tutor-responsable del caso y forma parte de la evaluación

Q4. Resolución del caso. Envío del informe final al tutor-responsable (semana 15 del semestre)

Al final del semestre, los grupos presentan la resolución del caso en clase ABP-FS (semana 16 del semestre)

Q1: análisis, ordenación y priorización de la información; Q2: investigación y 'lluvia de ideas' o formulación de hipótesis; Q3: solicitud de información clínica y exploraciones complementarias que puedan ayudar a argumentar la hipótesis; Q4: resolución. (previo al inicio del trabajo en grupo). En una segunda etapa, los integrantes del grupo de trabajo sintetizaban los conocimientos adquiridos y preparaban un informe con los datos clínicos analizados y las hipótesis surgidas del estudio de los casos, el cual debían publicar en la plataforma Moodle junto con una solicitud de datos clínicos y exploraciones complementarias que consideraban necesarios para verificar la hipótesis, argumentándola y señalando los resultados esperados y la interpretación de éstos (paso Q3).

El tutor responsable del caso, tras analizar la información comunicada por el grupo de trabajo, en el plazo de una semana remitía una valoración junto con los datos de historia clínica-anamnesis, exploración física y exploraciones complementarias solicitadas y correctamente argumentadas.

En el paso Q4, los alumnos, de forma grupal, elaboraban el producto final (informe o trabajo), presentando los resultados y el proceso seguido en la actividad. Dicho documento se publicaba en la plataforma Moodle para su posterior evaluación por parte del tutor-responsable del caso. Al final del semestre se programaron, por cada uno de los casos propuestos, dos sesiones presenciales, de dos horas cada una, para la presentación oral de los trabajos. En cada una de estas sesiones eran convocados cinco miembros de los grupos de trabajo, los cuales discutían públicamente entre ellos el análisis y resolución de los casos planteados, y eran evaluados por el profesor.

\section{Procedimiento de evaluación}

Como ya se ha mencionado, el ABP-FS se empleó también como un instrumento más de evaluación sumativa de los estudiantes de las asignaturas de 'Fisiopatogenia' y 'Semiología'. A continuación se detalla la distribución de las actividades de evaluación y su 'peso' porcentual en relación con la nota final en ambas asignaturas:

- Fisiopatogenia. Examen tipo test: 70\%; ABP: 10\%; ECOE: $20 \%$.

- Semiología. Examen tipo test: 70\%; ABP: 10\%; práctica clínica hospitalaria: $20 \%$.

Por lo que respecta al procedimiento de evaluación del ABP-FS, éste incluía la evaluación de los alumnos, tanto en el proceso como en el producto, y tanto individual como grupal. Para ello se preparó una hoja de cálculo con la ponderación de puntuaciones, atendiendo a los siguientes porcentajes: $30 \%$ de la nota del ABP para la información aportada a título individual Q1 y Q2 (información clínica analizada e 
Tabla II. Resultados de la asignatura de 'Fisiopatogenia' en los dos últimos cursos académicos.

\begin{tabular}{lcc}
\hline & Curso 2011/2012 & Curso 2012/2013 \\
\hline Matriculados & 76 & 74 \\
\hline Aprobados & 41 & 46 \\
\hline Notables & 3 & 11 \\
\hline Sobresalientes & 0 & 0 \\
\hline Suspendidos & 32 & 17 \\
\hline
\end{tabular}

hipótesis), 20\% para la información aportada a título grupal Q1 y Q2, 20\% para el apartado Q3 (solicitud argumentada de datos) y $30 \%$ para la presentación final de la resolución del ABP donde se analizaban, mediante un check-list diseñado a tal efecto, aspectos como el formato de la presentación, el contenido (conocimientos y enfoque), la presentación-transmisión de la información, la capacidad de síntesis y la valoración global del aprovechamiento.

Por otro lado, se realizó un estudio de la satisfacción de los estudiantes participantes en esta actividad de aprendizaje-enseñanza. El objetivo era valorar los aprendizajes adquiridos, preguntar sobre algunos aspectos positivos y negativos y determinar la carga real de trabajo que les había supuesto. Los docentes implicados también hicieron un cómputo de horas de dedicación.

\section{Resultados}

Durante el curso académico 2012-2013 participaron en esta actividad un total de 74 alumnos. La calificación media que los docentes asignaron al desarrollo de competencias propuestas con la actividad del ABP-FS fue de 7,3 puntos sobre 10.

A fin de examinar la utilidad de aplicar la metodología del ABP-FS, se analizaron los resultados obtenidos en las asignaturas de 'Fisiopatogenia' y 'Semiología' durante los dos últimos cursos académicos (curso 2011-2012, sin la aplicación del ABP como metodología de aprendizaje-enseñanza, y curso 2012-2013, en el que se aplicó el ABP). Atendiendo a los resultados obtenidos, hemos podido observar una mejora de éstos durante el curso 2012-2013 coincidiendo con la aplicación del ABP-FS (Tablas II y III). No obstante, no podemos afirmar que dicha mejora sea relacional, ya que no disponemos de
Tabla III. Resultados de la asignatura de 'Semiología' en los dos últimos cursos académicos.

\begin{tabular}{lcc}
\hline & Curso 2011/2012 & Curso 2012/2013 \\
\hline Matriculados & 75 & 74 \\
\hline Aprobados & 37 & 29 \\
\hline Notables & 9 & 38 \\
\hline Sobresalientes & 0 & 0 \\
\hline Suspendidos & 29 & 7 \\
\hline
\end{tabular}

datos que permitan atribuirla a la introducción del ABP.

En cuanto a la encuesta de satisfacción, fue respondida de forma anónima por 66 alumnos $(89,2 \%$ de respuesta).

Respecto al grado de satisfacción global obtenido tras la realización de la práctica del ABP-FS, el $74,6 \%$ señaló que era medio-alto. De acuerdo con la escala utilizada, los resultados quedan detallados en la figura.

En los resultados de la utilidad del ABP para el desarrollo del razonamiento clínico, se observa que el $87,3 \%$ percibió que la utilidad era buena-muy buena. Los datos detallados han sido los siguientes: el $46,3 \%(n=30)$, muy buena; el $41,8 \%(n=28)$, buena, y el 11,9\% $(n=8)$, normal/correcta.

En relación a si el ABP había sido de utilidad para el desarrollo de la capacidad de análisis, el $59,1 \%(n=39)$ manifestó estar muy de acuerdo/ completamente de acuerdo, un $36,4 \%(n=24)$ consideró la utilidad normal y un 4,5\% $(n=3)$ manifestó no estar de acuerdo en que fue útil a tal fin.

En referencia a la ayuda que había representado la realización del ABP para entender mejor las asignaturas de 'Fisiopatogenia' y 'Semiología', el 75,8\% de los encuestados manifestó 'bastante-mucho'. Los resultados detallados son los siguientes: el 59,1\% $(n=39)$, bastante; el 22,7\% $(n=15)$, normal; el $16,7 \%(n=11)$ señaló que les había ayudado mucho, y el $1,5 \%(n=1)$, poco.

Por último, en el cuestionario de satisfacción también se preguntó a los alumnos sobre si consideraban adecuado seguir manteniendo esta actividad en cursos posteriores. El 92\% lo consideró adecuado.

Desde un punto de vista cualitativo, los principales aspectos positivos manifestados por los alumnos respecto al ABP-FS fueron: 'se aprende a traba- 
Figura. Grado de satisfacción global del ABP en las asignaturas de 'Fisiopatogenia' y 'Semiología' percibido por los alumnos.

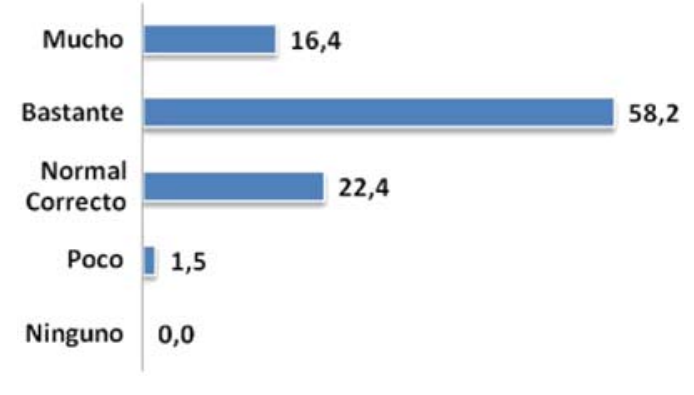

jar en grupo,' 'se aprende a relacionar contenidos teóricos con su aplicación', 'es de gran ayuda para el desarrollo de análisis clínico/razonamiento clínico' y 'es un aprendizaje más ameno'.

Como aspectos negativos o dificultades encontradas, los alumnos señalaron lo siguiente: 'el tiempo de dedicación que requiere', 'dificultad para reunir el grupo' y 'no tener suficiente experiencia con este tipo de metodología de ABP'.

La carga de trabajo para los docentes se limitó a una sesión presencial con todo los alumnos para mostrar la actividad de ABP-FS (2 horas), la asistencia y evaluación del resultado final del ABP asignado (uno por tutor, 4 horas) y las horas de tutorización y seguimiento virtual de la actividad (1,5 horas por cada uno de los 10 grupos de 7-8 alumnos).

\section{Discusión}

El ABP ha demostrado ser un instrumento válido para desarrollar competencias profesionales no abordadas por las metodologías clásicas. No obstante, existen importantes barreras para su implantación relacionadas con las dificultades de implementación en grupos con gran número de alumnos participantes, con el 'costo' (mayor dedicación en tiempo, por parte de los tutores) y con la coordinación en los programas de las asignaturas implicadas y la organización de los horarios, sobre todo de las sesiones presenciales con tutor y reuniones internas del grupo. No obstante, el modelo de ABP-FS implementado en el presente trabajo disminuye, en parte, las dificultades anteriormente mencionadas al favorecer la coordinación de profesores y asignaturas, al reducir la dedicación de carga de trabajo por la implicación de un equipo docente mayor (al estar involucradas dos asignaturas) y al disponer de una plataforma virtual que permite una gestión más ágil de las tutorías.

En lo referente al proceso de evaluación del ABPFS, se han encontrado algunas limitaciones: variabilidad en el grado de dificultad entre los problemas seleccionados como objeto de ABP y distinto nivel de formación teórica del tema del ABP en clase en el momento de preparar los casos. Asimismo, la evaluación del trabajo individual resulta difícil porque rápidamente se produce una comunicación entre los alumnos de los contenidos del razonamiento clínico, aspecto a mejorar en años sucesivos.

Como ventajas, el ABP-FS, según las observaciones de los alumnos, se ha mostrado útil para relacionar conocimientos de diferentes materias, ayuda a comprender mejor las asignaturas y permite trabajar competencias como el trabajo en equipo, el autoaprendizaje, etc. Esto podría explicar en parte los mejores resultados obtenidos en el curso 20122013, si bien nuestra impresión es que quizás en dicha mejora puede haber influido la motivación y el reto que suponía resolver los problemas planteados, junto al hecho de que dichos problemas eran muy transversales, lo que apoyaba un estudio de repaso más activo de las materias implicadas.

En conclusión, el modelo de ABP-FS ha mostrado ser aplicable en grandes grupos de alumnos y sin necesidad de recursos suplementarios, lo que permite hacer una primera valoración positiva respecto a su viabilidad. El empleo de plataformas virtuales puede ser un elemento clave para minimizar la carga de trabajo que conlleva la gestión del ABP.

Desde el punto de vista del aprendizaje logrado, los resultados evaluativos observados tras la implementación del ABP-FS son muy positivos.

Desde el punto de vista del alumno, el ABP-FS se ha considerado útil para el desarrollo de competencias específicas como el razonamiento clínico y de competencias trasversales como el trabajo en equipo.

Cabe señalar que la implementación de este tipo de metodologías activas no está exenta de obstáculos y requiere un esfuerzo adicional de alumnos y docentes, aunque a tenor de los resultados de satisfacción obtenidos, merece la pena.

Por último, consideramos que la metodología del ABP, con adaptaciones similares a las llevadas a cabo en esta experiencia, es aplicable desde los primeros cursos, si bien precisa una formación específica sobre el método tanto por parte de los docentes como por parte de los alumnos. 
Bibliografía

1. Ministerio de Educación, Cultura y Deporte. ¿Qué es el proceso de Bolonia? URL: http://www.educacion.gob.es/ boloniaensecundaria/01-que-es.htm. [01.04.2013].

2. Barrows HS, Tamblyn, RN. Problem-based learning: an approach to medical education. New York: Springer; 1980.

3. Barrows HS. A taxonomy of problem-based learning methods. Med Educ 1986; 20: 481-6.

4. Araújo UF, Sastre G. El aprendizaje basado en problemas. Una nueva perspectiva de la enseñanza en la universidad. Barcelona: Gedisa; 2008.

5. Lewis SE. La enseñanza basada en tópicos o problemas en la educación en ciencias. URL: http://www.actionbioscience. org/esp/education/lewis.html. [01.03.2006]

6. Learning-Theories.com. Problem-based learning (PBL) URL: http://www.learning-theories.com/problem-basedlearning-pbl.html. [01.04.2013].
7. Barrows HS. Problem-based learning in medicine and beyond: a brief overview. In Wilkerson L, Gijselaers WH, eds. Bringing problem-based learning to higher education: theory and practice. San Francisco: Jossey-Bass Publishers; 1996. p. 3-12.

8. Davis MH, Harden RM. AMEE Medical Education Guide no. 15. Problem-based learning: a practical guide. Med Teach 1999; 21: 130-40.

10. Koh GC, Khoo HE, Wong ML, Koh D. The effects of problembased learning during medical school on physician competency: a systematic review. CMAJ 2008; 178: 34-41.

10. Khoo HE. Implementation of problem-based learning in Asian medical schools and students' perceptions of their experience. Med Educ 2003; 37: 401-9.

11. Donner RS, Bickley H. Problem-based learning: an assessment of its feasibility and cost. Hum Pathol 1990; 21: 881-5. 